\title{
BMJ Open Protocol for intraoperative assessment of the human cerebrovascular glycocalyx
}

\author{
R H L Haeren, ${ }^{1} \mathrm{H}$ Vink, ${ }^{2}$ J Staals, ${ }^{3}$ M A M J van Zandvoort, ${ }^{4,5} \mathrm{~J}$ Dings, ${ }^{1,6}$ \\ J J van Overbeeke, ${ }^{1,6}$ G Hoogland, ${ }^{1,6}$ K Rijkers, ${ }^{1,7}$ O E M G Schijns ${ }^{1,6}$
}

To cite: Haeren RHL, Vink H, Staals J, et al. Protocol for intraoperative assessment of the human cerebrovascular glycocalyx. BMJ Open 2017;7:e013954.

doi:10.1136/bmjopen-2016013954

- Prepublication history for this paper is available online. To view these files please visit the journal online (http://dx.doi.org/10.1136/ bmjopen-2016-013954).

Received 19 August 2016 Revised 20 October 2016 Accepted 8 December 2016

CrossMark

For numbered affiliations see end of article.

Correspondence to

R H L Haeren;

roel.haeren@mumc.nl

\section{ABSTRACT}

Introduction: Adequate functioning of the blood-brain barrier (BBB) is important for brain homoeostasis and normal neuronal function. Disruption of the BBB has been described in several neurological diseases. Recent reports suggest that an increased permeability of the BBB also contributes to increased seizure susceptibility in patients with epilepsy. The endothelial glycocalyx is coating the luminal side of the endothelium and can be considered as the first barrier of the BBB. We hypothesise that an altered glycocalyx thickness plays a role in the aetiology of temporal lobe epilepsy (TLE), the most common type of epilepsy. Here, we propose a protocol that allows intraoperative assessment of the cerebrovascular glycocalyx thickness in patients with TLE and assess whether its thickness is decreased in patients with TLE when compared with controls.

Methods and analysis: This protocol is designed as a prospective observational case-control study in patients who undergo resective brain surgery as treatment for TLE. Control subjects are patients without a history of epileptic seizures, who undergo a craniotomy or burr hole surgery for other indications. Intraoperative glycocalyx thickness measurements of sublingual, cortical and hippocampal microcirculation are performed by video microscopy using sidestream dark-field imaging. Demographic details, seizure characteristics, epilepsy risk factors, intraoperative haemodynamic parameters and histopathological evaluation are additionally recorded.

Ethics and dissemination: This protocol has been ethically approved by the local medical ethical committee (ID: NL51594.068.14) and complies with the Declaration of Helsinki and principles of Good Clinical Practice. Informed consent is obtained before study enrolment and only coded data will be stored in a secured database, enabling an audit trail. Results will be submitted to international peer-reviewed journals and presented at international conferences.

Trial registration number: NTR5568.

\section{INTRODUCTION}

With a prevalence of 4-10 per 1000, epilepsy is one of the most common neurological disorders. ${ }^{1}$ The most frequent type of focal epilepsy is temporal lobe epilepsy (TLE). Although most patients have adequate seizure control by using antiepileptic drugs, it is estimated that $22.5-30 \%$ of patients are drug-resistant. ${ }^{2} 3$ This implies that worldwide about 13 million patients suffer from drug-refractory epilepsy, which has a major impact, both medically and socioeconomically. ${ }^{3} 4$ The development of new antiepileptic drugs has hardly reduced the number of drug-resistant patients, and therefore several different treatment alternatives have been explored over the past 25 years. Epilepsy surgery has been demonstrated to be a successful treatment alternative in selected patients. Furthermore, discovery of new drugs, targeted at specific underlying pathophysiologic mechanisms, keeps holding a promise for improved treatment of drug-resistant patients with epilepsy.

In this regard, important discoveries on microvasculature abnormalities in patients with TLE have been reported, such as loss of blood-brain barrier (BBB) integrity. ${ }^{5-7} \mathrm{~A} \mathrm{BBB}$ opening induces extravasation of proteins, like albumin, which in turn activates transforming growth factor $\beta$ (TGF $\beta$ ) signalling in astrocytes, leading to astrocytic transformation. ${ }^{7}$ This transformation is characterised by downregulation of inward rectifier potassium channels and astrocytic glutamate transporters, leading to impaired cerebral homoeostasis, altered neurovascular coupling, enhanced neuronal excitability and upregulation of proinflammatory cytokines. ${ }^{8} 9$ Moreover, leucocyte extravasation due to increased BBB permeability contributes to neuronal excitability and reorganisation of local neuronal networks. ${ }^{9}$ Thus, loss of BBB integrity results in increased seizure susceptibility and contributes to epileptogenesis.

The barrier function of the BBB is mainly determined by the endothelium. Endothelial cells are interconnected by tight junctions and adherent junctions to prevent paracellular 
diffusion. ${ }^{10}$ As a consequence, the endothelium forms a continuous cell membrane layer along the cerebral capillaries. Solutes and nutrients are transported by transport proteins expressed on the endothelial cells. ${ }^{10} 11$ Endothelial cells thus restrict and actively control the passage of substances from the blood to the brain in order to tightly regulate cerebral homoeostasis. The pericytes and astrocyte foot processes form a complex network surrounding the endothelial cells to induce and maintain endothelial barrier properties. As was recently pointed out, the endothelial glycocalyx could be a significant determinant of the BBB function as well. ${ }^{12}$ The endothelial glycocalyx, further referred to as 'glycocalyx', is a gel-like layer lining the luminal surface of the endothelium. It has important barrier properties that reduce the interaction between endothelial cells and plasma cells and components. As a consequence, the glycocalyx limits leucocyte adhesion and protein extravasation. ${ }^{13-16}$

The glycocalyx is a vulnerable layer that is easily disrupted, resulting in a reduced thickness. ${ }^{17}$ As part of the $\mathrm{BBB}$, a disrupted glycocalyx would result in increased BBB permeability and propagate leucocyte adhesion and extravasation. A number of laboratory and clinical studies have shown that both increased BBB permeability and leucocyte adhesion/extravasation play a role in epilepsy. $^{7-9} 18$

Until now, no data are available on glycocalyx thickness in epilepsy. The cerebrovascular glycocalyx has only been evaluated preclinically. ${ }^{19-22}$ None of these studies have analysed glycocalyx thickness in relation to neurological diseases. However, glycocalyx thickness can be assessed non-invasively as part of microcirculation imaging using sidestream dark-field (SDF) imaging. ${ }^{12}$ Until now, this technique has mainly been performed to assess the sublingual microcirculation.

Our hypothesis is that the cerebrovascular glycocalyx can be visualised clinically using SDF imaging, and that drug-resistant patients with TLE have a decreased cerebrovascular glycocalyx thickness in comparison to controls. In this paper, we present the study protocol according to the STROBE guidelines for case-control studies and SPIRIT protocol guidelines. ${ }^{23} 24$

The primary objective of this case-control study is to visualise the cerebrovascular glycocalyx using SDF imaging in patients with TLE and control patients during brain surgery. Moreover, an eventual correlation between the cerebrovascular and sublingual glycocalyx dimensions is assessed.

\section{METHODS}

\section{Study setting and population}

This is a multicentre prospective observational casecontrol study that involves assessment of the sublingual, cortical and hippocampal glycocalyx. In conjunction, data are recorded regarding the patients' medical history, current physiology and cerebral pathology. Assessments take place during brain surgery, which is performed by the Department of Neurosurgery of both the Maastricht University Medical Center+ (MUMC+) and the Zuyderland Medical Center (ZMC), respectively located in Maastricht and Heerlen, The Netherlands.

Patients with unilateral drug-resistant TLE who are designated for resective brain surgery, that is, anterior temporal lobectomy and/or amygdalohippocampectomy, are possible study candidates. The diagnosis of unilateral TLE is based on thorough examination including a video-EEG, high-resolution MRI, neuropsychological examination and, when indicated, a positron emission tomography scan and/or single-photon emission CT scan, functional MRI and eventually subdural or depth (stereo-EEG) electrode implantation. Subsequently, eligibility for resective surgery is assessed by a multidisciplinary team, consisting of neurologists, clinical neurophysiologists, neuropsychologists, neuroradiologists and neurosurgeons.

The control group includes patients without a history of epileptic seizures and those who will undergo a craniotomy for intracranial tumour resection or neurovascular indications, such as aneurysm clipping and arteriovenous malformation resection, or those who will undergo burr hole surgery for tumour biopsy.

We have included an upper age limit of 60 years to avoid 'background' microvascular disease which is strongly age-related. Since patients with tumours are generally already older, on average, than epilepsy patients, age could otherwise have been an important confounder. Control patients with vascular pathology, such as aneurysms and arteriovenous malformation, are not known to have microcirculatory pathology. Moreover, cortical measurements are performed at the furthest distance away of the vascular or oncological as allowed by the craniotomy. This could, by example, be the superior temporal gyrus in a patient with an anterior cerebral artery aneurysm or an inner ridge meningioma. Cortical measurements in the oncological control patients will also be performed at the furthest distance away from the abnormality as possible, as allowed by the craniotomy.

\section{Eligibility criteria}

Inclusion criteria are: mentally competent adults between 18 and 60 years of age who will undergo resective surgery for unilateral drug-resistant TLE, or for a tumour or vascular abnormality, or burr hole surgery for tumour biopsy (table 1). Exclusion from this study occurs in case of pregnancy, history of established hypertension, diabetes mellitus, hyperlipidaemia, stroke or other cardiovascular disease, use of cardiovascular medication, or nonsymptomatic signs of cerebral small vessel disease on brain MRI. Additionally, control patients in whom no 'normal', 'non-compressed' and/or 'non-edematous' cerebral cortex can be assessed during surgery or in whom a history of seizures is reported are excluded.

\section{Intervention protocol}

The glycocalyx thickness is assessed intraoperatively at the following time points: (1) a sublingual measurement 
Table 1 Overview of inclusion and exclusion criteria

\section{Inclusion criteria}

Patients with TLE

Mentally competent patients between 18 and 60 years of age.

Patients undergoing resective surgery as treatment of drug-resistant TLE with an epileptic focus, ie, located in a non-eloquent area.
Control patients

Mentally competent patients between 18 and 60 years of age.

Patients undergoing an elective craniotomy for intracranial tumour resection or neurovascular surgery, or undergoing burr hole surgery for tumour biopsy in a non-eloquent area.

\section{Exclusion criteria}

\section{Applicable to all patients}

Patients who are pregnant, who have been diagnosed for diabetes mellitus, hyperlipidaemia, stroke, or other cardiovascular diseases, or use of cardiovascular medication.

Patients who, based on brain MRI, show non-symptomatic signs of cerebral small vessel disease.

Applicable to control patients only

Patients in whom no 'normal', 'non-compressed' and/or 'non-edematous' cerebral cortex can be assessed intraoperatively.

Patients with a history of seizures.

TLE, temporal lobe epilepsy.

(M1) directly following the induction of general anaesthesia, (2) a cortical measurement (M2) on opening of the meninges allowing a direct view at the cortex and (3) a hippocampal measurement (M3) only in patients with TLE. The latter measurement is performed on removal of the temporal neocortex allowing a view of the hippocampus. At each time point, systolic and diastolic blood pressure, heart rate, pulse oxygen saturation, haemoglobin concentration and haematocrit are additionally recorded.

Glycocalyx measurements are performed using an SDF video microscope. The camera consists of a central light guide with a magnifying lens and concentric light emitting diodes. The diodes emit light at a wavelength of $530 \mathrm{~nm}$, which is absorbed by (de)-oxyhaemoglobin in erythrocytes. Consequently, erythrocytes appear black on a greyish background. This technique has mainly been performed on the sublingual microcirculation but has previously been used for cerebral microcirculation assessment as well. ${ }^{25-29}$ However, the glycocalyx was not measured in these studies. ${ }^{25-28}$ Recently, it was pointed out that SDF imaging, when combined with dedicated software, is the most suitable technique for clinical cerebrovascular glycocalyx visualisation. ${ }^{12}$

In order to reduce user-induced variations, image acquisition is trained. Using a camera with low pressure, minimising movement artefacts and optimising light intensity and focus are of particular importance to further reduce variability.

The glycocalyx analysis is based on the principle of the erythrocyte-endothelial exclusion zone. Since erythrocytes do not significantly compress the glycocalyx, there is an exclusion zone between the red blood cell column (RBCC) and the endothelium. GlycoCheck software automatically selects $\sim 3000$ microcirculatory vessel segments and measures the RBCC width at each segment. Based on the RBCC width distribution, the total perfused diameter (Dperf) is assessed by linear regression analysis of the 25th and 75th RBCC width percentiles. $^{12}$ The perfused boundary region (PBR) is the outermost luminal part of the glycocalyx that is only slightly permeable for erythrocytes. This region is calculated in a two-dimensional plane as follows: $\mathrm{PBR}=(\mathrm{Dperf}$ -median RBCC)/2. Since an unstable or damaged glycocalyx is more accessible to erythrocytes, an increased RBCC and, consequently, increased Dperf and PBR values signify a damaged glycocalyx. ${ }^{29}$

\section{Outcome}

The primary outcome of this study is cortical glycocalyx thickness in patients with TLE and controls. The thickness is estimated by measuring the PBR and the Dperf, both expressed in micrometres $(\mu \mathrm{m})$.

As a secondary outcome, sublingual, cortical and hippocampal glycocalyx thickness in patients with TLE and controls are evaluated within and between groups. In addition, we aim to analyse the relation between these measures and epilepsy-specific risk factors (eg, febrile seizures, traumatic brain injury), seizure characteristics, drug use (eg, type of drugs, dosage) and histopathological outcome (eg, degree of hippocampal sclerosis, type of cortical dysplasia). Finally, glycocalyx thickness values will be correlated to general demographic (eg, age, gender, bodyweight, smoking status) and intraoperative clinical parameters.

\section{Recruitment capacity, consent and timeline}

We have calculated a sample size of 15 patients for each group, to be included over 4 years. At the MUMC+ and $\mathrm{ZMC}$, all types of surgery required for inclusion are performed at least 15-20 times every year. Based on these surgery numbers, the likelihood to recruit the calculated sample size is high.

The study participants' timeline is outlined below and an overview is also given in figure 1 . 


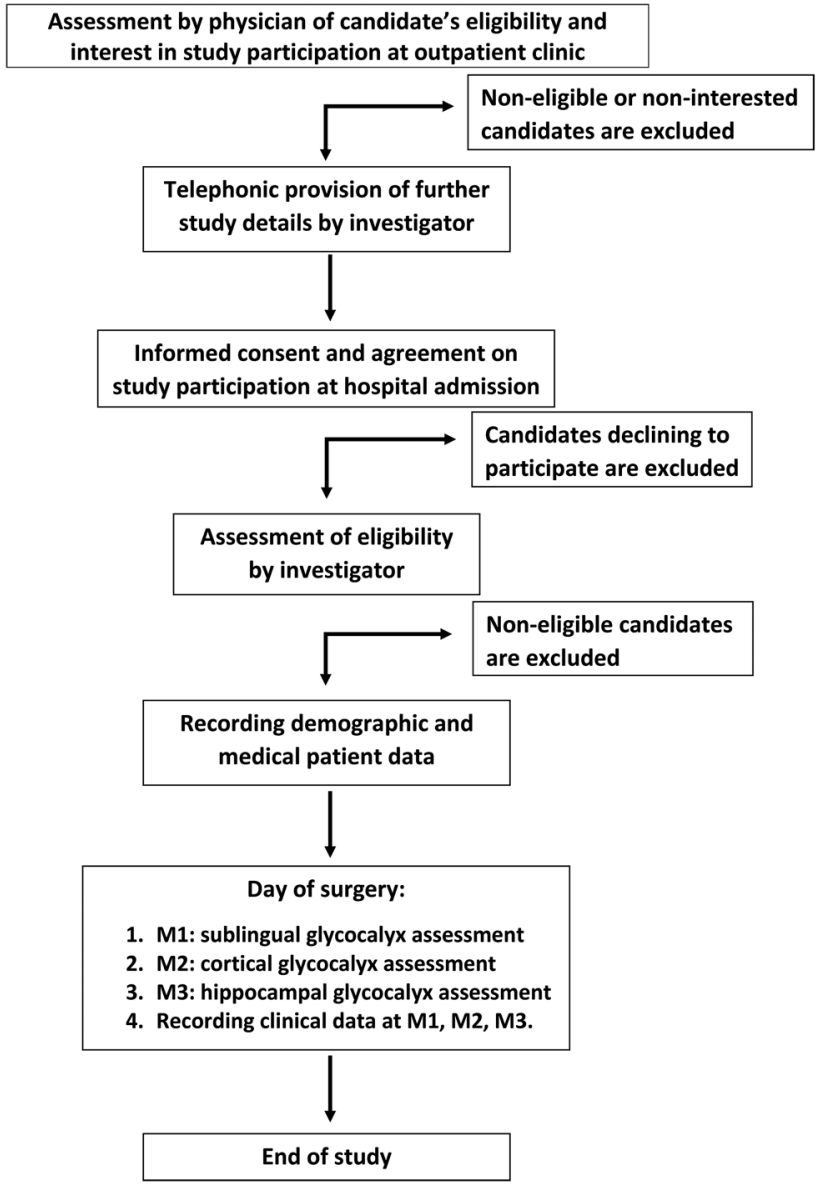

Figure 1 Overview of inclusion and exclusion criteria.

\section{Recruitment}

Patients will visit the neurosurgical outpatient clinic preoperatively. During this visit, the neurosurgeon will ask the patients whether they are interested to take part in the study. If interested, they will receive the applicable patient information brochure (PIB). Within a few days, possible candidates will be informed more extensively by one of the investigators about the study objective, procedures, benefits, risks and insurance. Participation decisions will be inquired on the day of admission to the hospital. Patients are allowed at least 3 days to consider participation in the study, starting from the moment of receiving the PIB. Participation is voluntarily and does not affect standard treatment in any way. Patients will be informed that they can decide to end their participation in the study at any time.

2. Informed consent

On the day of hospital admission, the patients' study participation is ultimately inquired. When patients agree on participation, the informed consent agreement will be signed in duplicate.

3. Data recording

Following informed consent agreement, patient data will be recorded from the digital patient files. The digital patient files encompass the digital patient dossier at the
MUMC+, ZMC and, when applicable, the file of the multidisciplinary epilepsy surgery workup.

4. Intraoperative measurements

A total of two or three glycocalyx measurements will be performed, as described in the Intervention protocol section. All measurements are performed at the operating theatre when the patient is under general anaesthesia. The hippocampal measurement is solely performed in patients with TLE.

5. End of study

Directly following the final measurements, that is, M2 in control patients and M3 in patients with TLE, patients have reached the end point of this study.

\section{Population size}

We have calculated a population size of 15 patients per group. This number is based on a power of $80 \%$ to detect a difference of cortical glycocalyx thickness of at least $12 \%$ between groups with an SD of $15 \%$, at a significance level of 0.05 and an expected drop-out of two patients.

Since there is no literature on cerebrovascular glycocalyx thickness, we have reviewed and assessed the literature on variation in sublingual glycocalyx measurements. Intraindividual variation of sublingual glycocalyx thickness, assessed using SDF imaging, has been found to be $\pm 5 \% .{ }^{30}$ A difference in PBR thickness of $9.6-12.5 \%$ with an SD of $\pm 15 \%$ has been found relevant when comparing a disease state to healthy controls. ${ }^{29-32}$ On the basis of these studies, we have determined minimal glycocalyx difference and SD. Owing to the explorative nature of our study, clinical relevance of a $12 \%$ difference is indistinct.

\section{Data processing \\ Procedures}

This study complies with the Declaration of Helsinki, and will be conducted in accordance with the principles of Good Clinical Practice (GCP). Standardised processing files for obtaining informed consent, measurement procedures, reporting (serious) adverse events (AEs), and recording patient and measurement data parameters in the electronic case report file (eCRF) are available. Investigators obtaining informed consent from the patient, performing glycocalyx measurements and recording eCRF data will receive specific training beforehand.

\section{Data management}

Patients' demographic and clinical data are recorded in an eCRF at a secure encrypted database (by Castor Electronic Data Capture (EDC)), which enables an audit trail and is GCP certified. Measurement procedures are trained and standardised as described in the Intervention protocol section. Measurement data are collected at a secure encrypted laptop and outcome is recorded in the eCRF. After verification of recorded 
data to source data by one of the executive investigators, recorded data in the eCRF by Castor EDC will be exported to an SPSS file for further statistical analysis.

Patients will be assigned an alphanumeric sequential study number to identify all clinical data. Patients' demographic data linked to the assigned study number are documented in a separate screening database held on a secure computer at both study sites. Source data, the code encrypting document and coded data in the study database are locked and only accessible to the principal and executive researchers and monitors. On completion of the study, the study database will be locked and data are securely archived for 15 years in accordance with local policy.

Owing to the nature and short participation time period of this study, we expect full patient retention and adherence.

\section{Safety}

The principal investigator (OEMGS) has overall responsibility for the conduct of this study including safety. Individual investigators will be responsible for reporting AEs and serious AEs (SAEs) to the principal investigator. SAEs are defined as AEs resulting in death, lifethreatening events, prolonged hospital stays or significant disability. There are no reported (S)AEs associated with the use of SDF imaging in the current literature. A possible attributable risk of glycocalyx measurement is sublingual, cortical or hippocampal contusion due to pressure on the tissue during the measurement. We deem risk frequency and severity as low. Moreover, cortical or hippocampal local contusion will take place in non-eloquent and to-be-resected tissue. Postoperative consequences due to this contusion are unlikely. All events are reviewed by the principal investigator to decide if there is a causal link and, when applicable, appropriate action will be undertaken. SAEs will be reported to the local medical ethical committee (METC azM/UM) according to local policy. Liability and subject insurance are provided.

\section{Statistical analysis}

Data are presented as mean and SD when normally distributed, and non-normally distributed data as median and IQR. Glycocalyx dimensions between groups are compared using the independent Student's t-test or the Mann-Whitney test, as appropriate. Correlation between sublingual and cortical, sublingual/cortical and hippocampal glycocalyx thickness is calculated by Pearson's or Spearman's correlation coefficient, when data are normally or non-normally distributed, respectively. In patients with TLE, the cortical and hippocampal glycocalyx thickness results are correlated to seizure characteristics, epilepsy risk factors, anti-epileptic drug usage and histological findings of pathological examination by using Pearson's or Spearman's correlation coefficient or univariate and multivariate regression analysis, as appropriate. Demographic and clinical parameters are correlated to glycocalyx thickness results by using univariate and multivariate regression analysis. A $p$ value of $<0.05$ will be considered statistically significant. Statistical analysis is performed using SPSS software.

Interim analyses will be performed yearly and following data recording of five patients in each group. When a significant difference between the groups regarding the primary outcome is found, the study will be terminated prior to inclusion of 15 patients in each group. Interim analyses are reported to the local medical ethical committee.

\section{Monitoring and auditing}

This study was classified as minimal risk by the local data monitoring committee (Clinical Trial Center Maastricht (CTCM)). Monitoring visits include a review of consent and study procedures according to study protocols, source data and audit trail verification, and the review of (serious) AE reporting. Monitoring is independent and performed at least once a year. Monitor evaluations are reported to the local medical ethical committee. Unannounced audits can be performed by the audit team of the CTCM.

\section{Ethics, amendments and dissemination}

This research protocol has been approved by the local medical ethical committee (Medisch-ethische toetsingscommissie academisch ziekenhuis Maastricht/Maastricht University (METC azM/UM)) and has been assigned the following protocol ID: NL51594.068.14. Also, this study has been registered at the Netherlands National Trial Register (ID: NTR5568). The NTR is acknowledged by the WHO and International Committee of Medical Journal Editors (ICMJE).

Substantive protocol amendments will be reported, reviewed and approved by the METC azM/UM before application. In the currently presented protocol, a variety of substantive protocol amendments have already been incorporated. Substantive amendments were introduced due to lagging inclusion. The substantive amendments included extending the study by the addition of the ZMC as a study centre, enlarging the control group with burr hole tumour biopsy and patients with neurovascular surgery, and reducing sublingual measurements from three to one.

Results of this study will be evaluated yearly and following five participants in both groups. Results will be recorded using audit trails to increase reproducibility. Study protocol and results will be submitted to peer-reviewed journals and presented at international conferences.

\section{DISCUSSION}

Microvascular injury, in particular increased permeability of the wall of the cerebral microcirculation, seems to play a role in the transformation of astrocytes and increased seizure susceptibility in epilepsy. It is hypothesised that leakage of albumin orchestrates this transformation leading to a disturbed potassium and glutamate 
metabolism and extracellular cerebral homoeostasis. ${ }^{7} 8$ Upregulation of proinflammatory cytokines as a response to leakage of leucocytes further contributes to the subsequent increased neuronal excitability. ${ }^{9}$ However, it is still unclear why some patients do and some patients do not develop epilepsy following a first seizure. Heinemann et al have brought up the hypothesis that lasting dysfunction of the cerebral microcirculation results in dysregulation of the normal network response to injury, leading to the development of epilepsy. Therefore, they have emphasised the urgent need for non-invasive clinical visualisation of the cerebral microcirculation in order to evaluate local dynamical functioning and possible injury. ${ }^{8}$

The glycocalyx is a significant component of the cerebral microcirculation and involved in the regulation of plasma cell adhesion, oxidative stress and shear stress reduction. ${ }^{12} 13$ The glycocalyx is easily disrupted, that is, reduced in thickness. ${ }^{17}$ Disruption is induced by local cytokine expression and ischaemia, and results in inflammation, oedema, oxidative stress and loss of vascular responsiveness. ${ }^{12}$ Also, many vascular disease risk factors and specific diseases such as lacunar stroke, sepsis and renal failure are associated with decreased glycocalyx thickness. ${ }^{29} 33-36$

The glycocalyx plays an important role in vascular wall permeability. Albumin extravasation is prohibited by the negatively charged glycocalyx, whereas leucocytes cannot attach to endothelial surface components including important cell-adhesion molecules, such as intercellular adhesion molecule 1 (ICAM-1) and vascular cell adhesion molecule 1 (VCAM-1), that reside within the glycocalyx. As a consequence, leucocyte adhesion and extravasation is limited. Therefore, a disturbed glycocalyx is suspected to play a significant role in increased BBB permeability including albumin and leucocyte extravasation, as is seen in epilepsy.

Assessment of the cerebrovascular glycocalyx offers the opportunity to gain greater insights into its thickness and function at the level of the cerebral microcirculation. Advances in microcirculation imaging by hand-held SDF-based video microscopes enable real-time clinical assessment of the microcirculation. Several cerebral microcirculation parameters, but not the glycocalyx, have been evaluated using this technique. ${ }^{25-28}$ It was recently pointed out that SDF imaging is the most eligible technique when proper software is subjoined. ${ }^{12}$ Using SDF imaging, we aim to visualise the cerebrovascular glycocalyx and measure its thickness in patients with TLE and controls. Subsequently, cerebrovascular glycocalyx dimensions are compared with sublingual dimensions to establish whether glycocalyx dimensions are regulated at a systemic level.

In this study, we will determine whether glycocalyx thickness is reduced in patients with TLE when compared with controls. The included patients with TLE have undergone a thorough examination and were selected by a multidisciplinary team. Naturally, genuinely healthy controls for intracranial cerebrovascular glycocalyx assessment are not available, making the selected patients the most suitable candidates as controls. Since an intact haemodynamic circulation is required for SDF imaging, postmortem patients cannot be included as controls.

The upper age cut-off of 60 years is included to reduce the influence of microcirculatory degeneration which is associated with increasing age. Since patients with tumours are, on average, older patients than patients with epilepsy, this could otherwise have been an important confounder. In order to limit the risk of vascular abnormalities of the visualised and assessed vessels, patients will only be included as controls when a noncompressed and/or non-oedematous cerebral cortex can be assessed during surgery. In the control group, the cortical measurements are performed at the furthest distance away from the abnormality, as is allowed by the craniotomy.

Typical pathological findings in TLE include hippocampal sclerosis characterised by neuronal cell loss, reactive astrogliosis, mossy fibre sprouting and granular cell dispersion. ${ }^{5}$ Unfortunately, assessment of the hippocampal glycocalyx can only be performed in patients with TLE, as the hippocampus will rarely be exposed in the control subjects. Hippocampal glycocalyx thickness of patients with TLE will be compared with the cortical glycocalyx thickness and cannot be compared with hippocampal controls.

There are some important variables that could affect the glycocalyx thickness that are not directly assessed in this study. These variables concern haemodynamic variations, intravascular volume variations and the effect of different anaesthetics. Owing to the explorative nature of this study, we have not included these variables, although post-hoc analyses will be performed to determine confounding. Another reason for exclusion of these variables is that this would necessitate additional preoperative (awake) and intraoperative sublingual measurements of the glycocalyx. At first, these measurements were included in our study protocol. However, owing to anxiety for the preoperative measurement, and the risks for sterility of the surgery due to the intraoperative measurements, these sublingual measurements were excluded in an amendment (see the Ethics, amendments, and dissemination section). The possible effects of intraoperative haemodynamic variation and anaesthesia on glycocalyx thickness could, however, be an interesting future study. When clinically relevant differences in glycocalyx dimensions are found, future research to determine glycocalyx component variation, glycocalyx permeability determinants and mechanisms of disruption and repair is required. Subsequently, repair of the glycocalyx could be a selective and efficient, yet hypothetical, target for modification of increased BBB permeability. This would open a new field of pharmacological interventions for currently drug-resistant patients with epilepsy. 
Author affiliations

${ }^{1}$ Department of Neurosurgery, School for Mental Health and Neuroscience, Maastricht University Medical Center, Maastricht, The Netherlands ${ }^{2}$ Department of Physiology, Cardiovascular Research Institute Maastricht, Maastricht University, Maastricht, The Netherlands

${ }^{3}$ Department of Neurology, Cardiovascular Research Institute Maastricht, Maastricht University Medical Center, Maastricht, The Netherlands ${ }^{4}$ Department of Genetics \& Cell Biology, Cardiovascular Research Institute Maastricht, Maastricht University, Maastricht, The Netherlands

${ }^{5}$ Institute for Molecular Cardiovascular Research, IMCAR, Universitätsklinikum, Aachen University, Aachen, Germany

${ }^{6}$ Academic Center for Epileptology, Maastricht University Medical Center and Kempenhaeghe, Maastricht/Heeze, The Netherlands

${ }^{7}$ Department of Neurosurgery, Zuyderland Medical Center, Heerlen, The Netherlands

Contributors RHLH acted as an executive investigator and contributed to the concept, drafting, design and revising of the protocol. HV contributed to the concept and critically revising of the protocol. JS contributed to the design and critically revising of the protocol. MAMJvZ contributed to the concept and critically revising of the protocol. JD contributed to the concept and design of the protocol. JJv0 contributed to the design and critically revising of the protocol. GH contributed to the concept and design of the protocol. KR acted as an executive investigator, and contributed to the concept, drafting and design of the protocol. OEMGS acted as an principal investigator, and contributed to the concept, design and critically revising of protocol.

Competing interests HV is the Chief Science Officer at GlycoCheck BV.

Ethics approval Medical Ethical Committe academisch ziekenhuis Maastricht/ Maastricht University.

Provenance and peer review Not commissioned; externally peer reviewed.

Data sharing statement The original protocol and substantive amendments are available. These were available for the included authors and the local medical ethical committee.

Open Access This is an Open Access article distributed in accordance with the Creative Commons Attribution Non Commercial (CC BY-NC 4.0) license, which permits others to distribute, remix, adapt, build upon this work noncommercially, and license their derivative works on different terms, provided the original work is properly cited and the use is non-commercial. See: http:// creativecommons.org/licenses/by-nc/4.0/

\section{REFERENCES}

1. Forsgren L, Beghi E, Oun A, et al. The epidemiology of epilepsy in Europe-a systematic review. Eur J Neurol 2005;12:245-53.

2. Picot MC, Baldy-Moulinier M, Daurès JP, et al. The prevalence of epilepsy and pharmacoresistant epilepsy in adults: a population-based study in a Western European country. Epilepsia 2008:49:1230-8.

3. Laxer KD, Trinka E, Hirsch LJ, et al. The consequences of refractory epilepsy and its treatment. Epilepsy Behav 2014;37:59-70.

4. Jennum P, Gyllenborg J, Kjellberg J. The social and economic consequences of epilepsy: a controlled national study. Epilepsia 2011;52:949-56.

5. Thom M. Review: hippocampal sclerosis in epilepsy: a neuropathology review. Neuropathol Appl Neurobiol 2014:40:520-43.

6. Mott RT, Thore CR, Moody DM, et al. Reduced ratio of afferent to total vascular density in mesial temporal sclerosis. J Neuropathol Exp Neurol 2009;68:1147-54.

7. van Vliet EA, Aronica E, Gorter JA. Role of blood-brain barrier in temporal lobe epilepsy and pharmacoresistance. Neuroscience 2014:277:455-73.

8. Heinemann U, Kaufer D, Friedman A. Blood-brain barrier dysfunction, TGF $\beta$ signaling, and astrocyte dysfunction in epilepsy. Glia 2012;60:1251-7.

9. Vezzani A, Friedman A, Dingledine RJ. The role of inflammation in epileptogenesis. Neuropharmacology 2013;69:16-24.

10. Abbott NJ, Patabendige AAK, Dolman DEM, et al. Structure and function of the blood-brain barrier. Neurobiol Dis 2010;37:13-25
11. Ballabh $P$, Braun $A$, Nedergaard $M$. The blood-brain barrier: an overview. Neurobiol Dis 2004:16:1-13.

12. Haeren RHL, van de Ven SEM, van Zandvoort MAMJ, et al. Assessment and imaging of the cerebrovascular glycocalyx. Curr Neurovasc Res 2016;13:249-60.

13. Reitsma S, Slaaf DW, Vink H, et al. The endothelial glycocalyx: composition, functions, and visualization. Pflugers Arch Eur J Physiol 2007;454:345-59.

14. Curry FE, Adamson RH. Endothelial glycocalyx: permeability barrier and mechanosensor. Ann Biomed Eng 2012;40:828-39.

15. Constantinescu AA, Vink H, Spaan JE. Endothelial cell glycocalyx modulates immobilization of leukocytes at the endothelial surface. Arterioscler Thromb Vasc Biol 2003;23:1541-7.

16. Rops AL, van den Hoven MJ, Baselmans MM, et al. Heparan sulfate domains on cultured activated glomerular endothelial cells mediate leukocyte trafficking. Kidney Int 2008;73:52-62.

17. Reitsma S, Oude Egbrink MGA, Vink $\mathrm{H}$, et al. Endothelial glycocalyx structure in the intact carotid artery: a two-photon laser scanning microscopy study. J Vasc Res 2011;48:297-306.

18. Friedman A. Blood-brain barrier dysfunction, status epilepticus, seizures, and epilepsy: a puzzle of a chicken and egg? Epilepsia 2011;52(Suppl 8):19-20.

19. Mazzetti S, Librizzi L, Frigerio S, et al. Molecular anatomy of the cerebral microvessels in the isolated Guinea-pig brain. Brain Res 2004;999:81-90.

20. Hempel C, Hyttel P, Kurtzhals JAL. Endothelial glycocalyx on brain endothelial cells is lost in experimental cerebral malaria. $J$ Cereb Blood Flow Metab 2014;34:1107-10.

21. Sillesen M, Rasmussen LS, Jin G, et al. Assessment of coagulopathy, endothelial injury, and inflammation after traumatic brain injury and hemorrhage in a porcine model. $J$ Trauma Acute Care Surg 2014;76:12-19; discussion 19-20.

22. Jepsen $\mathrm{CH}$, DeMoya MA, Perner A, et al. Effect of valproic acid and injury on lesion size and endothelial glycocalyx shedding in a rodent model of isolated traumatic brain injury. $J$ Trauma Acute Care Surg 2014:77:292-7.

23. von Elm E, Altman DG, Egger M, et al. The Strengthening the Reporting of Observational Studies in Epidemiology (STROBE) statement: explanation and Elaboration. J Clin Epidemiol 2008:61:344-9.

24. Chan AW, Tetzlaff JM, Altman DG, et al. SPIRIT 2013 statement: defining standard protocol items for clinical trials. Ann Intern Med 2013;158:200-7.

25. Pérez-Bárcena $\mathrm{J}$, Goedhart $\mathrm{P}$, Ibáñez $\mathrm{J}$, et al. Direct observation of human microcirculation during decompressive craniectomy after stroke. Crit Care Med 2011;39:1126-9.

26. Pérez-Bárcena J, Romay E, Llompart-Pou JA, et al. Direct observation during surgery shows preservation of cerebral microcirculation in patients with traumatic brain injury. J Neurol Sci 2015:353:38-43.

27. Wan Z, Ristagno G, Sun S, et al. Preserved cerebral microcirculation during cardiogenic shock. Crit Care Med 2009;37:2333-7.

28. Šitina $M$, Turek $Z$, Pařzková $R$, et al. In situ assessment of the brain microcirculation in mechanically-ventilated rabbits using sidestream dark-field (SDF) imaging. Physiol Res 2011;60:75-81.

29. Martens RJH, Vink $\mathrm{H}$, Van Oostenbrugge RJ, et al. Sublingual microvascular glycocalyx dimensions in lacunar stroke patients. Cerebrovasc Dis 2013;35:451-4

30. Nieuwdorp M, Meuwese MC, Mooij HL, et al. Measuring endothelial glycocalyx dimensions in humans: a potential novel tool to monitor vascular vulnerability. J Appl Physiol 2008;104:845-52.

31. Snoeijs MG, Vink $H$, Voesten $N$, et al. Acute ischemic injury to the renal microvasculature in human kidney transplantation. Am J Physiol Renal Physiol 2010;299:F1134-40.

32. Dane MJC, Khairoun MR, Hyun Lee D, et al. Association of kidney function with changes in the endothelial surface layer. Clin J Am Soc Nephrol 2014:9:698-704.

33. Vlahu CA, Lemkes BA, Struijk DG, et al. Damage of the endothelial glycocalyx in dialysis patients. J Am Soc Nephrol 2012;23:1900-8.

34. Becker BF, Chappell D, Jacob M. Endothelial glycocalyx and coronary vascular permeability: the fringe benefit. Basic Res Cardiol 2010;105:687-701.

35. Ostrowski SR, Berg RMG, Windeløv NA, et al. Coagulopathy catecholamines, and biomarkers of endothelial damage in experimental human endotoxemia and in patients with severe sepsis: a prospective study. J Crit Care 2013;28:586-96.

36. Singh A, Fridén V, Dasgupta I, et al. High glucose causes dysfunction of the human glomerular endothelial glycocalyx. Am J Physiol Renal Physiol 2011;300:F40-8. 